\title{
Approximate Solution of an Infectious Disease Model Applying Homotopy Perturbation Method
}

\author{
Terhemen Simon Atindiga ${ }^{1}$, Ezike Godwin $\mathrm{Mbah}^{2}$, Ndidiamaka Edith Didigwu ${ }^{2}$, \\ Adebisi Raphael Adewoye ${ }^{3}$ \& Torkuma Bartholomew Kper ${ }^{4}$
}

${ }^{1}$ Department of Basic Sciences, Akperan Orshi College of Agriculture, Yandev, Gboko, Benue State, Nigeria

${ }^{2}$ Department of Mathematics, University of Nigeria, Nsukka, Enugu State, Nigeria

${ }^{3}$ Department of Maths. And Stats., Rufus Giwa Polytechnic, Owo, Ondo State, Nigeria

${ }^{4}$ ICT Directorate, Akperan Orshi College of Agriculture, Yandev, Gboko, Benue State, Nigeria

Correspondence: Terhemen Simon Atindiga, Department of Basic Sciences, Akperan Orshi College of Agriculture, Yandev, Gboko, Benue State, Nigeria.

Received: December 11, 2019 Accepted: March 25, 2020 Online Published: April 20, 2020

doi:10.5539/gjhs.v12n5p64

URL: https://doi.org/10.5539/gjhs.v12n5p64

\begin{abstract}
Scientists and engineers have developed the use of Homotopy Perturbation Method (HPM) in non-linear problems since this approach constantly distort the intricate problem being considered into a simple problem, thus making it much less complex to solve. The homotopy perturbation method was initially put forward by He (1999) with further development and improvement (He 2000a, He, 2006). Homotopy, which is as an essential aspect of differential topology involves a coupling of the conventional perturbation method and the homotopy method in topology (He, 2000b). The approach gives an approximate analytical result in series form and has been effectively applied by various academia for various physical systems namely; bifurcation, asymptotology, nonlinear wave equations and Approximate Solution of SIR Infectious Disease Model (Abubakar et al., 2013).
\end{abstract}

Keywords: approximate solution, infectious disease model, homotopy perturbation method

\section{Model Equations}

Considering the following systems of non-linear ordinary differential equation given as;

$\frac{d S}{d t}=b+a_{1} S_{v c}-\alpha_{1} c S-\mu S$

$\frac{d S_{v}}{d t}=\alpha_{1} c S-(1-\varphi) S_{v}-\mu S_{v}$

$\frac{d S_{v c}}{d t}=(1-\varphi) S_{v}-\left(1-e_{1}\right) \lambda S_{v c}-a_{1} S_{v c}-q S_{v c}-\mu S_{v c}$

$\frac{d S_{v c r}}{d t}=q S_{v c}-(1-e) \lambda S_{v c r}-\mu S_{v c r}$

$\frac{d S_{v e}}{d t}=\left(1-e_{1}\right) \lambda S_{v c}+(1-e) \lambda S_{v c r}-\rho_{2} S_{v e}-\mu S_{v e}$

$\frac{d I}{d t}=\rho_{2} S_{v e}-(1-\gamma) I-\mu I$

$\frac{d I_{t}}{d t}=(1-\gamma) I-d_{2} I_{t}-\mu I_{t}$

We let, 
$g_{1}=\left(\alpha_{1} c+\mu\right), \quad g_{2}=(1-\varphi), \quad g_{3}=\left(g_{2}+\mu\right)$,

$g_{4}=\left(1-e_{1}\right) \lambda, \quad g_{5}=\left(g_{4}+a_{1}+q+\mu\right), g_{6}=(1-e) \lambda, \quad g_{7}=\left(g_{6}+\mu\right)$,

$g_{8}=\left(\rho_{2}+\mu\right), \quad g_{9}=(1-\gamma), \quad g_{10}=(1-\gamma+\mu), g_{11}=\left(d_{2}+\mu\right)$

Rewriting (1) in a more compact form, we obtain;

$\frac{d S}{d t}=b+a_{1} S_{v c}-g_{1} S$

$\frac{d S_{v}}{d t}=\alpha_{1} c S-g_{3} S_{v}$

$\frac{d S_{v c}}{d t}=g_{2} S_{v}-g_{5} S_{v c}$

$\frac{d S_{v c r}}{d t}=q S_{v c}-g_{7} S_{v c r}$

$\frac{d S_{v e}}{d t}=g_{4} S_{v c}+g_{6} S_{v c r}-g_{8} S_{v e}$

$\frac{d I}{d t}=\rho_{2} S_{v e}-g_{10} I$

$\frac{d I_{t}}{d t}=g_{9} I-g_{11} I_{t}$

\section{Basic Idea of He's Homotopy Perturbation Method}

To demonstrate the basic idea of He's homotopy perturbation method, we consider the non linear differential equation, [He, 2000].

$$
A(u)-f(r)=0 \quad r \in \Omega
$$

Subject to the boundary condition of:

$$
B\left(u, \frac{\partial u}{\partial n}\right)=0, \quad r \in \Gamma
$$

Given that;

$A$ : the general differential operator,

$B$ : the boundary operator

$f(r)$; a known analytical solution and

$\Gamma$ : the boundary of the domain $\Omega$, Taghipour, (2011)

The general operator, A can be divided into two parts viz; $\mathrm{L}$ and $\mathrm{N}$ in which $\mathrm{L}$ is the linear part and the nonlinear part being N. Hence (3) will now become;

$$
L(u)+N(u)-f(r)=0 \quad r \in \Omega
$$

We shall now construct a homotopy $V(r, p)$ such that

$V(r, p): \Omega \times[0,1] \rightarrow R$ satisfing that;

$P \in[0,1], r \in \Omega$

$$
H(r, p)=(1-p)\left[L(v)-L\left(u_{0}\right)\right]+p[L(v)+N(v)-f(r)]=0
$$

Or

$$
H(r, p)=L(v)-L\left(u_{0}\right)+p L\left(u_{0}\right)+[N(v)-f(r)]=0
$$

Where 
$L(u)$ is the linear part

$L(u)=L(v)-L\left(u_{0}\right)+p L\left(u_{0}\right)$ and $N(u)$ is the non-linear term.

$N(u)=p N(v)$

$P \in[0,1]$ is an embedding parameter, while $u_{0}$ is an initial approximation of equation (3) which satisfies the boundary conditions.

Obviously, considering equations(6) and (7), we have

$$
\begin{aligned}
H(v, 0)=L(v)-L\left(u_{0}\right) & =0 \\
H(v, 1)=A(v)-f(r) & =0
\end{aligned}
$$

The changing process of $p$ from zero to unity is just that of $V(r, p)$ from $u_{0}$ to $u(r)$. In topology, this is called deformation while $L(v)-L\left(u_{0}\right), A(v)-f(r)$ are called homotopy.

According to Homotopy perturbation method (HPM), we can first use the embedding parameter, $p$ as a small parameter and assume solution for equation (6) and (7) which can be expressed as;

$$
V=v_{0}+p v_{1}+p^{2} v_{2}+\cdots
$$

If we let $p=$ unity, an approximate solution of equation (10) can be obtained as;

$$
U=\lim _{p \rightarrow 1} v=v_{0}+v_{1}+v_{2}+\cdots
$$

Equation (11) is the analytical solution of (3) by homotopy perturbation method.

He (2003), (2006) makes the following suggestion for convergence of (11)

(a). The second derivative of $N(v)$ wrt $V$ must be small because parameter, $p$ must be relatively large i.e $p \rightarrow 1$

(b). The norm of $L^{-1} \frac{\partial N}{\partial V}$ must be smaller than one so that the series converge.

We now apply HPM on the system (3) by assuming the solution as;

$$
\begin{aligned}
& S=u_{0}+P u_{1}+P^{2} u_{2}+\cdots \\
& S_{v}=w_{0}+P w_{1}+P^{2} w_{2}+\cdots \\
& S_{v c}=x_{0}+P x_{1}+P^{2} x_{2}+\cdots \\
& S_{v c r}=y_{0}+P y_{1}+P^{2} y_{2}+\cdots \\
& S_{v e}=z_{0}+P z_{1}+P^{2} z_{2}+\cdots \\
& I=m_{0}+P m_{1}+P^{2} m_{2}+\cdots \\
& I_{t}=n_{0}+P n_{1}+P^{2} n_{2}+\cdots
\end{aligned}
$$

From the the first equation of (12),

$\frac{d S}{d t}=b+a_{1} S_{v c}-g_{1} S$

The linear part is

$\frac{d s}{d t}=0$

and the non-linear part is

$$
b+a_{1} S_{v c}-g_{1} S=0
$$

We now apply HPM

$\Rightarrow(1-P) \frac{d S}{d t}+P\left[\frac{d S}{d t}-b-a_{1} S_{v c}+g_{1} S\right]=0$

Expanding, this gives

$\frac{d S}{d t}-P \frac{d S}{d t}+P \frac{d S}{d t}-P\left(b+a_{1} S_{v c}-g_{1} S\right)=0$ 
$\Rightarrow \frac{d S}{d t}-P\left(b+a_{1} S_{v c}-g_{1} S\right)=0$

$\Rightarrow \frac{d S}{d t}-P b-P a_{1} S_{v c}+P g_{1} S=0$

Substituting the first and third equations of (12) into (13) gives

$\left(u_{0}^{\prime}+P u_{1}^{\prime}+P^{2} u_{2}^{\prime}+\cdots+\right)-P b-P a_{1}\left(x_{0}+P x_{1}+P^{2} x_{2}+\cdots\right)$

$+P g_{1}\left(u_{0}+P u_{1}+P^{2} u_{2}+\cdots\right)=0$

Collecting the coefficient of powers of $P$, we have;

$P^{0}: u_{0}^{\prime}=0$

$P^{1}: u_{1}^{\prime}-b-a_{1} x_{0}+g_{1} u_{0}=0$

$P^{2}: u_{2}^{\prime}-a_{1} x_{1}+g_{1} u_{1}=0$

Applying the same approach, we have the following ;

$P^{0}: w_{0}^{\prime}=0$

$P^{1}: w_{1}^{\prime}-\alpha_{1} c u_{0}+g_{3} w_{0}=0$

$P^{2}: w_{2}^{\prime}-\alpha_{1} c u_{1}+g_{3} w_{1}=0$

$P^{0}: x_{0}^{\prime}=0$

$P^{1}: x_{1}^{\prime}-g_{2} w_{0}+g_{5} x_{0}=0$

$P^{2}: x_{2}^{\prime}-g_{2} w_{1}+g_{5} x_{1}=0$

$P^{0}: y_{0}^{\prime}=0$

$P^{1}: y_{1}^{\prime}-q x_{0}+g_{7} y_{0}=0$

$P^{2}: y_{2}^{\prime}-q x_{1}+g_{7} y_{1}=0$

$P^{0}: z_{0}^{\prime}=0$

$P^{1}: z_{1}^{\prime}-g_{4} x_{0}-g_{6} y_{0}+g_{8} z_{0}=0$

$P^{2}: z_{2}^{\prime}-g_{4} x_{1}-g_{6} y_{1}+g_{8} z_{1}=0$

$P^{0}: m_{0}^{\prime}=0$

$P^{1}: m_{1}^{\prime}-\rho_{2} z_{0}+g_{10} m_{0}=0$

$P^{2}: m_{2}^{\prime}-\rho_{2} z_{1}+m_{1} g_{10}=0$

$P^{0}: n_{0}^{\prime}=0$

$P^{1}: n_{1}^{\prime}-g_{9} m_{0}+g_{119} n_{0}=0$

$P^{2}: n_{2}^{\prime}-g_{9} m_{1}+g_{11} n_{1}=0$

From the first equation of (14),

$u_{0}^{\prime}=0$ 
$\frac{d u_{0}}{d t}=0$

$\Rightarrow d u_{0}=0$

Integrating gives us

$\int d u_{0}=S_{0}$

$\therefore u_{0}=c_{0}$

Where $c_{0}$ is constant of integration. Applying the initial condition we have

$u_{0}(0)=S_{0}$

$\Rightarrow c_{0}=S_{0}$

$\therefore u_{0}=S_{0}$

Similarly, we have that;

$\therefore S_{v 0}=w_{0}$

$\therefore S_{v c 0}=x_{0}$

$\therefore S_{v c r 0}=y_{0}$

$\therefore S_{v e 0}=z_{0}$

$\therefore I_{0}=m_{0}$

$\therefore I_{t_{0}}=n_{0}$

From the second equation of (14),

$u_{1}^{\prime}-b-a_{1} x_{0}+g_{1} u_{0}=0$,

$u_{1}^{\prime}=b+a_{1} x_{0}-g_{1} u_{0}$

$\Rightarrow \frac{d u_{1}}{d t}=b+a_{1} x_{0}-g_{1} u_{0}$

$\Rightarrow d u_{1}=\left(b+a_{1} x_{0}-g_{1} u_{0}\right) d t$

Substituting the first and third equations of the system (21) into (22) we obtain;

$d u_{1}=\left(b+a_{1} S_{v c 0}-g_{1} S_{0}\right) d t$

Integrating with respect to $t$, we have;

$u_{1}=\left(b+a_{1} S_{v c 0}-g_{1} S_{0}\right) t+c_{7}$

Where $c_{7}$ is constant of integration. Applying the initial condition we have;

$u_{1}(0)=0, \quad \Rightarrow c_{7}=0$

$\therefore u_{1}=\left(b+a_{1} S_{v c 0}-g_{1} S_{0}\right) t$ 
Similarly, we have that;

$\therefore w_{1}=\left(\alpha_{1} c S_{0}-g_{3} S_{v 0}\right) t$

$\therefore x_{1}=\left(g_{2} S_{v 0}-g_{5} S_{v c 0}\right) t$

$\therefore y_{1}=\left(q S_{v c 0}-g_{7} S_{v c r 0}\right) t$

$\therefore z_{1}=\left(g_{4} S_{v c 0}+g_{6} S_{v c r 0}-g_{8} S_{v e 0}\right) t$

$\therefore m_{1}=\left(\rho_{2} S_{v e 0}-g_{10} I_{0}\right) t$

$\therefore n_{1}=\left(g_{9} I_{0}-g_{119} I_{t 0}\right) t$

From the third equation of (14),

$u_{2}^{\prime}-a_{1} x_{1}+g_{1} u_{1}=0$

$u_{2}^{\prime}=a_{1} x_{1}-g_{1} u_{1}$

$\Rightarrow \frac{d u_{2}}{d t}=a_{1} x_{1}-g_{1} u_{1}$

$\Rightarrow d u_{2}=\left(a_{1} x_{1}-g_{1} u_{1}\right) d t$

Substituting the first and third equations of (23) into (24) we obtain;

$d u_{2}=\left[a_{1}\left(g_{2} S_{v 0}-g_{5} S_{v c 0}\right) t-g_{1}\left(b+a_{1} S_{v c 0}-g_{1} S_{0}\right) t\right] d t$

$d u_{2}=\left[a_{1}\left(g_{2} S_{v 0}-g_{5} S_{v c 0}\right)-g_{1}\left(b+a_{1} S_{v c 0}-g_{1} S_{0}\right)\right] t d t$

$d u_{2}=\left[-b g_{1}-\left(a_{1} g_{1}+a_{1} g_{5}\right) s_{v c 0}+a_{1} g_{2} S_{v 0}+g_{1}^{2} S_{0}\right] t d t$

Integrating both sides with respect to $t$, we have;

$u_{2}=\left[-b g_{1}-\left(a_{1} g_{1}+a_{1} g_{5}\right) s_{v c 0}+a_{1} g_{2} S_{v 0}+g_{1}^{2} S_{0}\right] \frac{t^{2}}{2}+c_{14}$

Where $c_{14}$ is constant of integration. Applying the initial condition we have;

$u_{2}(0)=0, \quad \Rightarrow c_{14}=0$

$\therefore u_{2}=\left[-b g_{1}-\left(a_{1} g_{1}+a_{1} g_{5}\right) s_{v c 0}+a_{1} g_{2} S_{v 0}+g_{1}^{2} S_{0}\right] \frac{t^{2}}{2}$

Similarly, we have that;

$$
\begin{aligned}
& \therefore w_{2}=\left[\alpha_{1} b c-\left(\alpha_{1} c g_{1}+\alpha_{1} c g_{3}\right) s_{0}+a_{1} \alpha_{1} c S_{v c 0}+g_{3}^{2} S_{v 0}\right] \frac{t^{2}}{2} \\
& \therefore x_{2}=\left[\alpha_{1} g_{2} c S_{0}-\left(g_{2} g_{3}+g_{2} g_{5}\right) S_{v 0}+g_{5}^{2} S_{v c 0}\right] \frac{t^{2}}{2} \\
& \therefore y_{2}=\left[q g_{2} S_{v 0}-\left(q g_{5}+q g_{7}\right) S_{v c 0}+g_{7}^{2} S_{v c r 0}\right] \frac{t^{2}}{2} \\
& \therefore z_{2}=\left[g_{2} g_{4} S_{v 0}-\left(g_{4} g_{5}+g_{4} g_{8}-q g_{6}\right) S_{v c 0}-\left(g_{6} g_{7}+g_{6} g_{8}\right) S_{v c r 0}+g_{8}^{2} S_{v e 0}\right] \frac{t^{2}}{2} \\
& \therefore m_{2}=\left[\rho_{2} g_{4} S_{v c 0}+\rho_{2} g_{6} S_{v c r 0}-\left(\rho_{2} g_{8}+\rho_{2} g_{10}\right) S_{v e 0}+g_{10}^{2} I_{0}\right] \frac{t^{2}}{2} \\
& \therefore n_{2}=\left[\rho_{2} g_{9} S_{v e 0}-\left(g_{9} g_{10}+g_{9} g_{11}\right) I_{0}+g_{11}^{2} I_{t 0}\right] \frac{t^{2}}{2}
\end{aligned}
$$

Substituting the first equations of (21), (23) and (25) into the number one equation of system (12), we 
obtain;

$S(t)=$

$S_{0}+P\left(b+a_{1} S_{v c 0}-g_{1} S_{0}\right) t+P^{2}\left[-b g_{1}-\left(a_{1} g_{1}+a_{1} g_{5}\right) s_{v c 0}+a_{1} g_{2} S_{v 0}+g_{1}^{2} S_{0}\right] \frac{t^{2}}{2}+\cdots$

Setting $p=1$, we obtain;

$$
\left.\begin{array}{l}
S(t)=S_{0}+\left(b+a_{1} S_{v c 0}-g_{1} S_{0}\right) t+\left[-b g_{1}-\left(a_{1} g_{1}+a_{1} g_{5}\right) s_{v c 0}+a_{1} g_{2} S_{v 0}+g_{1}^{2} S_{0}\right] \frac{t^{2}}{2}+\cdots \\
S_{v}(t)=S_{v 0}+\left(\alpha_{1} c S_{0}-g_{3} S_{v 0}\right) t+\left[\alpha_{1} b c-\left(\alpha_{1} c g_{1}+\alpha_{1} c g_{3}\right) s_{0}+a_{1} \alpha_{1} c S_{v c 0}+g_{3}^{2} S_{v 0}\right] \frac{t^{2}}{2}+\cdots \\
S_{v c}(t)=S_{v c 0}+\left(g_{2} S_{v 0}-g_{5} S_{v c 0}\right) t+\left[\alpha_{1} g_{2} c S_{0}-\left(g_{2} g_{3}+g_{2} g_{5}\right) S_{v 0}+g_{5}^{2} S_{v c 0}\right] \frac{t^{2}}{2}+\cdots \\
S_{v c r}(t)=S_{v c r 0}+\left(q S_{v c 0}-g_{7} S_{v c r 0}\right) t+\left[q g_{2} S_{v 0}-\left(q g_{5}+q g_{7}\right) S_{v c 0}+g_{7}^{2} S_{v c r 0}\right] \frac{t^{2}}{2}+\cdots \\
S_{v e}(t)=S_{v e 0}+\left(g_{4} S_{v c 0}+g_{6} S_{v c r 0}-g_{8} S_{v e 0}\right) t+\left[g_{2} g_{4} S_{v 0}-\left(g_{4} g_{5}+g_{4} g_{8}-q g_{6}\right) S_{v c 0}-\left(g_{6} g_{7}+\right.\right.
\end{array}\right\}
$$

Hence, equations (45) to (51) are our model equations in HPM.

\section{Conclusion}

In this paper, we solved some nonlinear time dependent ordinary differential equations analyticall to obtain approximate solutions using Homotopy Perturbation Method. We considered a system of nonlinear ordinary differential equations arising from the developed mathematical model of an infectious disease. We applied He's same approach in handling the model equations when applying Homotopy Perturbation Method (HPM) to obtain approximate solutions. The result shows the efficiency of homotopy perturbation method in solving nonlinear equations.

\section{Competing Interests Statement}

The authors declare that there are no competing or potential conflicts of interest.

\section{References}

Abubakar, S., Akinwande, N. I., Jimoh, O. R., Oguntolu, F. A., \& Ogwumu, O. D. (2013). Approximate Solution of SIR Infectious Disease Model Using Homotopy Perturbation Method (HPM). Pacific Journal of Science and Technology, 14(2), 163-169.

Ganji, D. D., \& Rajabi, A. (2006). Assessment of homotopy-perturbation and perturbation methods in heat radiation equations. International Communications in Heat and Mass Transfer, 33(3), 391-400. https://doi.org/10.1016/j.icheatmasstransfer.2005.11.001

Ganji, D. D., \& Sadighi, A. (2006). Application of He's homotopy-perturbation method to nonlinear coupled systems of reaction-diffusion equations. International Journal of Nonlinear Sciences and Numerical Simulation, 7(4), 411-418. https://doi.org/10.1515/IJNSNS.2006.7.4.411

He, J. H. (1999). Homotopy perturbation technique, computer methods in applied mechanics and engineering. International J nonlinear mechanics, 35, 37-43. https://doi.org/10.1016/S0045-7825(99)00018-3

He, J. H. (2000a). A coupling method of homotopy technique and perturbation to Volterra's integro-differential equation. Int. J. Non-Linear Mech, 35(1), 37-43. https://doi.org/10.1016/S0020-7462(98)00085-7 
He, J. H. (2000b). A new perturbation technique which is also valid for large parameters. Journal of Sound and Vibration, 229(5), 1257-1263. https://doi.org/10.1006/jsvi.1999.2509

He, J. H. (2006). Addendum: new interpretation of homotopy perturbation method. International journal of modern physics B, 20(18), 2561-2568. https://doi.org/10.1142/S0217979206034819

Taghipour, R. (2011). Application of homotopy perturbation method on some linear and nonlinear periodic equations. World Appl Sci J, 10(10), 1232-1235.

\section{Copyrights}

Copyright for this article is retained by the author(s), with first publication rights granted to the journal.

This is an open-access article distributed under the terms and conditions of the Creative Commons Attribution license (http://creativecommons.org/licenses/by/4.0/). 